\title{
Direct Iliac Vein Stenting in Phlegmasia Cerulea Dolens Caused by May-Thurner Syndrome
}

\author{
Nikolaos Galanakis ${ }^{1}$, Nikolaos Kontopodis ${ }^{2}$, Elias Kehagias ${ }^{1}$, Nikolaos Daskalakis ${ }^{2}$, Konstantinos \\ Tsetis ${ }^{1}$, Christos V. loannou ${ }^{2}$, and Dimitrios Tsetis ${ }^{1}$ \\ ${ }^{1}$ Interventional Radiology Unit, Department of Medical Imaging, ${ }^{2}$ Vascular Surgery Unit, Department of Cardiothoracic and Vascular \\ Surgery, University Hospital of Heraklion, University of Crete Medical School, Heraklion, Greece
}

\begin{abstract}
Phlegmasia cerulea dolens (PCD) is an uncommon but potentially limb-threatening complication of acute deep vein thrombosis. A 56-year-old male presented with PCD. Color Doppler ultrasonography revealed extensive thrombosis of the left superficial and common femoral and external and common iliac veins. After an unsuccessful iliofemoral thrombectomy, contrast-enhanced computed tomography revealed iliofemoral deep vein thrombosis due to May-Thurner syndrome (MTS). After the deployment of an inferior vena cava filter, the thrombotic occlusion was traversed with a guidewire and direct stenting was performed to achieve immediate recanalization. The patient was discharged two days after the procedure, demonstrating significant clinical improvement. MTS is a rare cause of PCD. Direct iliac vein stenting may be a safe and effective alternative treatment for rapid recanalization if percutaneous mechanical thrombectomy devices are unavailable.
\end{abstract}

Key Words: Phlegmasia cerulea dolens, May-Thurner syndrome, Deep venous thrombosis, Direct stenting
Received April 19, 2021

Revised September 19, 2021

Accepted December 3, 2021

Published on December 27, 2021

Corresponding author: Nikolaos Galanakis Interventional Radiology Unit, Department of Medical lmaging, University Hospital Heraklion, University of Crete Medical School, Lili Zografou 4, Heraklion 71307, Greece

Tel: 30-2810-392033

Fax: 30-2810-392460

E-mail: nikos_meduoc@hotmail.com https://orcid.org/0000-0001-9878-6053

Copyright (c) 2021 The Korean Society for Vascular Surgery

This is an Open Access article distributed under the terms of the Creative Commons Attribution Non-Commercial License (http://creativecommons.org/licenses/by-nc/4.0) which permits unrestricted non-commercial use, distribution, and reproduction in any medium, provided the original work is properly cited.

Cite this article; Vasc Specialist Int 2021. https://doi.org/10.5758/vsi.210031

\section{INTRODUCTION}

Phlegmasia cerulea dolens (PCD) is a rare but limbthreatening complication of acute deep vein thrombosis (DVT) that is characterized by extensive swelling, severe pain, and cyanosis. PCD is associated with massive fluid sequestration, which can lead to hypovolemia and may progress to limb ischemia and venous gangrene [1]. Early diagnosis and proper treatment are crucial for the management of PCD, as it is characterized by increased mortality and major amputation rate, especially in cases with progression to venous gangrene [2]. The risk factors for PCD include malignancy, heparin-induced thrombocytopenia, antiphospholipid syndrome, surgery, femoral vein catheterization, contraceptive agent use, heart failure, and pregnancy [3], but malignancy and hypercoagulable states are the most common causes [2]. May-Thurner syndrome (MTS) is caused by extrinsic compression of the left common iliac vein against the lumbar vertebra by the overlying right common iliac artery. MTS is associated with impedance of the iliac vein outflow and ipsilateral DVT, but it rarely progresses to PCD. Its prevalence is unknown; however, it is found in $2 \%$ to $3 \%$ of patients with lower-extremity DVT $[4,5]$. MTS is more common in females (two fold higher), but males present with a more significant degree of pain and swelling, whereas females are more likely to have pulmonary embolism in addition to DVT on presentation [6]. PCD requires immediate blood flow restoration to limit the progression of gangrenous changes in patients with profound venous outflow obstruction. Here we present a case of PCD caused by MTS that was successfully treated with direct iliac vein stenting. This case report was approved by 
the Institutional Review Board (no. 1088581215), and written informed consent was obtained from the patient for publication of this article.

\section{CASE}

A 56-year-old male presented to the emergency department with a 24-hour history of left lower limb pain, extensive swelling and cyanosis (Fig. 1A). His medical history was unremarkable except for cigarette smoking (52 packyears) and chronic obstructive pulmonary disease. He did not take any medications regularly and had no history of malignancy, recent travel, trauma or falling. Color Doppler ultrasonography (CDUS) revealed extensive thrombosis of the left superficial femoral, common femoral, external iliac, and common iliac veins. Femoral, popliteal and ankle arterial pulses were undetectable. The patient was initially transferred to the operating theater, where an emergency left iliofemoral thrombectomy was attempted. First, the left common femoral vein was prepared using a longitudinal skin incision at the groin. A venotomy was performed at the level of the saphenofemoral junction. The infrainguinal thrombus was successfully removed by elevation and tight wrapping of the leg with a rubber bandage, followed by compression of the calf and thigh with the foot at a dorsiflexed position. After the infrainguinal thrombus removal, a proximal thrombectomy was performed. However, it was unsuccessful because the thrombectomy catheter could not be advanced $20 \mathrm{~cm}$ beyond the femoral incision. Additionally, although a large amount of clot was retrieved in each attempt, the thrombus seemed to form again and the congestion of the limb did not improve.

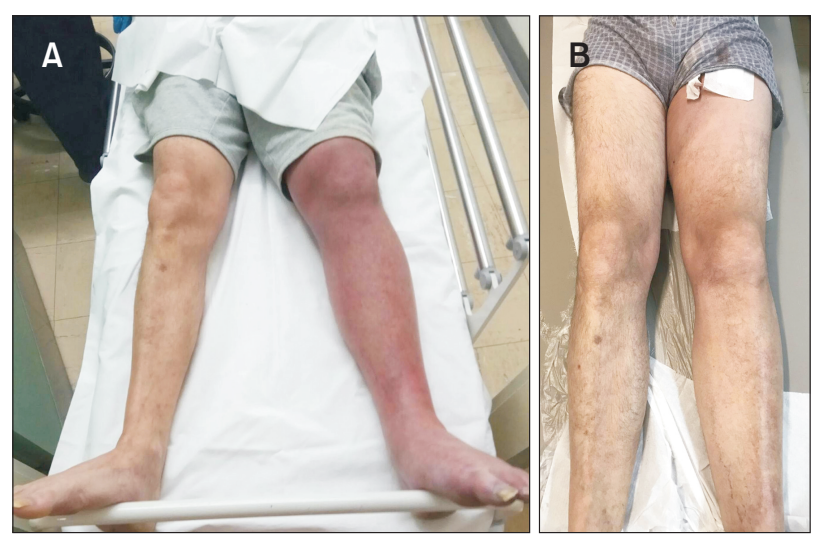

Fig. 1. Photographs of the patient's limbs. (A) At the time of presentation, the left limb was painful and swollen with a bluish-red discoloration. (B) Two days after endovascular revascularization, significant improvement was noted and the patient's symptoms resolved.
Subsequent contrast-enhanced computed tomography revealed the presence of MTS (Fig. 2). In the angiographic suite, both common femoral veins were punctured under ultrasound guidance and a left extremity venography showed complete thrombosis of the left external and common iliac veins (Fig. 3A). The occlusion was traversed using a stiff 0.035-in guidewire (Terumo, Tokyo, Japan) and a Bern catheter (Boston Scientific, Natick, MA, USA) (Fig. 3B). A Denali inferior vena cava filter (IVCF; Bard Peripheral Vascular Inc., Tempe, AZ, USA) was placed through the right common femoral vein to prevent a pulmonary embolism, which was deployed at the level of the $\mathrm{L} 3$ vertebra because of the presence of a retroaortic left renal vein at that point. Due to unavailability of mechanical thrombectomy devices, direct stenting was performed to achieve immediate recanalization and flow restoration. Two sinusvenous stents (OptiMed $\mathrm{GmbH}$, Ettlingen, Germany), an 18 $\mathrm{mm} \times 80 \mathrm{~mm}$ and an $18 \mathrm{~mm} \times 60 \mathrm{~mm}$, were deployed with a $10-\mathrm{mm}$ overlap across the occluded segments. The proximal stent was placed first. Stent deployment was followed by post-dilatation using a slightly undersized percutaneous transluminal angioplasty (PTA) balloon catheter, an ATLAS $16 \mathrm{~mm} \times 60 \mathrm{~mm}$ (Bard Peripheral Vascular lnc.) at low pressures to avoid laceration and dislodgment of the thrombus. The final digital subtraction angiography (DSA) revealed significant flow restoration (Fig. 3C). There were no clinical signs of pulmonary embolism during the procedure.

After the procedure, the patient's limb pain was significantly improved and the peripheral pulses were restored. Subsequently, the swelling and cyanosis were relieved (Fig.

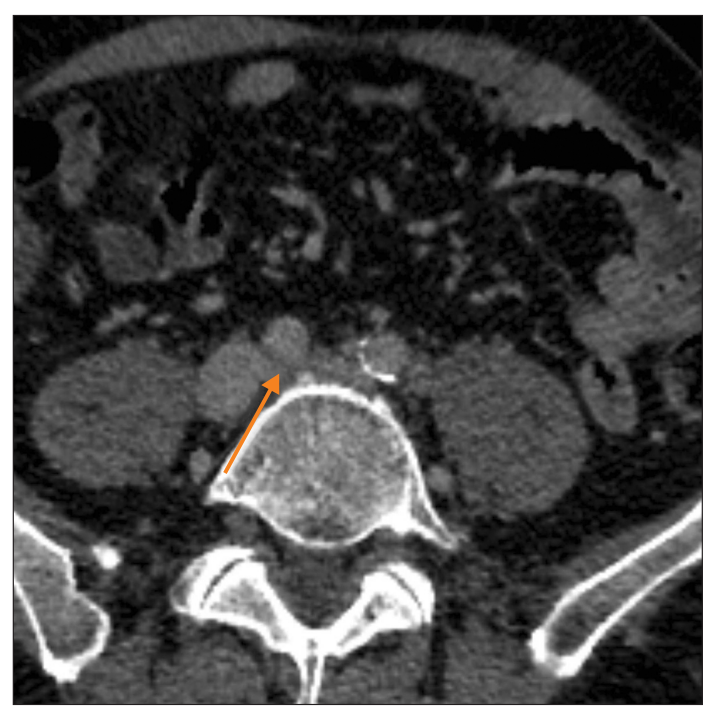

Fig. 2. Computed tomography image showed the left common iliac vein compressed by the right common iliac artery (arrow). 


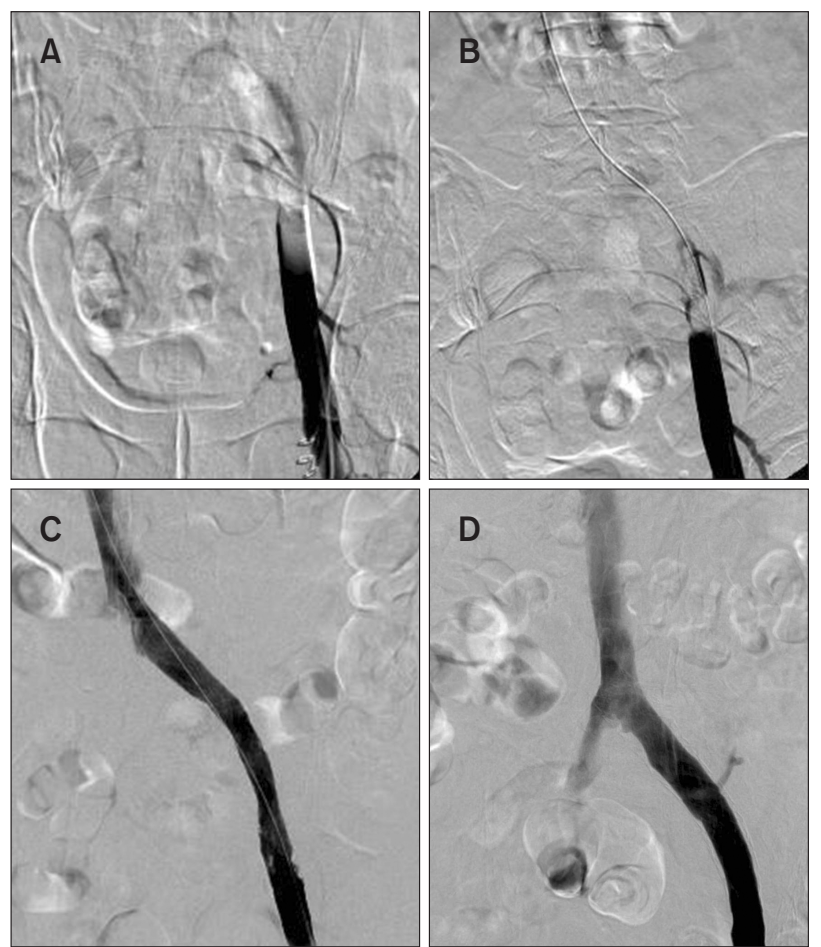

Fig. 3. (A) Digital subtraction angiography (DSA) showed thrombotic occlusion of the left common and external iliac veins. (B) The occluded segment was traversed with a guidewire. (C) Final DSA showed significant flow restoration after the deployment of two overlapping venous stents. (D) Three months later, the inferior vena cava filter was removed and DSA revealed excellent stent patency without signs of thrombosis or restenosis.

$1 \mathrm{~B})$ and the patient was discharged 3 days later on longterm oral anticoagulation with rivaroxaban. The patient was evaluated for malignancy or hypercoagulable states, but all laboratory and imaging examinations were negative. After 3 months, the IVCF was removed and DSA showed excellent stent patency without restenosis or thrombosis (Fig. 3D). During the 6-month follow-up, there were no clinical signs of pulmonary embolism and CDUS showed no evidence of recurrent DVT.

\section{DISCUSSION}

The management of PCD to prevent serious complications such as venous gangrene and tissue loss includes surgical thrombectomy, catheter-directed thrombolysis (CDT) and percutaneous mechanical thrombectomy (PMT). Anticoagulation, fluid resuscitation and leg elevation constitute the initial management of the disease, but this approach is ineffective in severe cases of PCD [7]. Surgical thrombectomy provides rapid revascularization and prevents throm- bus propagation and gangrene development. However, it is associated with high rate of recurrent thrombosis and cannot prevent venous stasis caused by valvular incompetence. Moreover, it requires general anesthesia and may be unsuccessful in cases of severe venous stenosis as in MTS [2]. CDT is a safe and effective treatment for patients with lower-extremity DVT, as it is associated with high rate of thrombus clearance and reduced rate of long-term postthrombotic syndrome (PTS) [8]. However, prolonged time may be required to achieve flow restoration [9]. PMT is an effective treatment for early thrombus removal with high technical success which can be also used as an adjunct to CDT $[7,10]$. However, PMT devices are not always available.

lliocaval stenting is indicated in patients with nonthrombotic obstructive venous lesions with $>30 \%$ stenosis. Moreover, it can be considered an adjunct to interventional or surgical management of iliocaval thrombosis, but it is rarely used as a primary treatment for PCD [11]. Stenting of chronic iliocaval venous obstruction is characterized by low morbidity and mortality, high long-term patency rate and low restenosis rate [12]. Venous stenting for DVT was first described by Berger et al. [13], who reported the case of a patient with MTS who was successfully treated with CDT and stent placement after an insufficient response to PTA. Since then, stenting has emerged as an effective treatment option for MTS. A recent multicenter study demonstrated that stenting in MTS is associated with good short- and long-term outcomes, long-term patency and a low risk for PTS [14]. However, there are only a few case reports of patients with PCD caused by MTS $[15,16]$. To the best of our knowledge, this is the first case of successful endovascular treatment using direct iliac vein stenting for MTS-induced PCD. Direct stenting using self-expanding nitinol stents can seal off the thrombus, providing rapid flow restoration and avoiding laceration and dislodgment of thrombus. This technique has been used in cases of acute arterial occlusions with excellent technical and clinical success and a low risk of distal embolization [17]. Thus, it would be effective in cases of unavailability of PMT devices. Regarding proper stent selection, venous dedicated stents are recommended in iliofemoral venous occlusive disease. Arterial stents usually have smaller diameter and high radial force, which affect thin venous wall patency and are associated with high restenosis rate. On the other hand, venous dedicated stents are characterized by proper diameter, flexibility, crush resistance, radial strength and the ability to allow precise and accurate deployment and adapt the venous shape [18]. Several studies have shown that venous dedicated stents present excellent technical results with low morbidity and high patency rates [19]. Moreover, venous dedicated stents demonstrated improved primary, assisted primary and second- 
ary patencies in patients with chronic iliofemoral obstruction suffering from PTS [20]. Pre-dilatation is generally performed in patients with chronic occlusions. However, in acute DVT, it may lead to dislodgment of the thrombus and pulmonary embolism; thus, direct stenting, which can seal off the thrombus, is a safer approach. It is also important that the proximal and distal ends of the stents lie in the healthy venous segment. If multiple stents are used, they must be deployed in an overlapping manner without skipped areas. Moreover, in cases of MTS, stents may need to be exceeded into the inferior vena cava to avoid early restenosis. On the other hand, stenting across the inguinal ligament should be avoided to prevent in-stent restenosis. However, stenting into a normal flow segment is more important [9]. Finally, routine placement of an IVCF is not indicated during thrombus removal procedures (surgical or endovascular), but we believe it was necessary in this case because of the risk of clot dislodgement and pulmonary embolism.

In conclusion, MTS is an uncommon cause of PCD and should be suspected in patients without a history of malignancy, surgery or hematologic disease. Direct iliac vein stenting may be a safe and effective alternative treatment option for rapid revascularization in patients with PCD caused by MTS if PMT devices are unavailable.

\section{FUNDING}

None.

\section{CONFLICT OF INTEREST}

The authors have nothing to disclose.

\section{ORCID}

\author{
Nikolaos Galanakis \\ https://orcid.org/0000-0001-9878-6053 \\ Nikolaos Kontopodis \\ https://orcid.org/0000-0002-6792-5003 \\ Elias Kehagias \\ https://orcid.org/0000-0002-2537-2893 \\ Nikolaos Daskalakis \\ https://orcid.org/0000-0001-5385-4294 \\ Konstantinos Tsetis \\ https://orcid.org/0000-0001-9561-7588 \\ Christos V. loannou \\ https://orcid.org/0000-0002-9929-6184 \\ Dimitrios Tsetis \\ https://orcid.org/0000-0002-6648-6117
}

\section{AUTHOR CONTRIBUTIONS}

Concept and design: NG, NK. Analysis and interpretation: NG, EK. Data collection: NG, ND. Writing the article: NG, KT. Critical revision of the article: NK, CVI, DT. Final approval of the article: all authors. Statistical analysis: NG. Obtained funding: none. Overall responsibility: NG, DT.
1) Perkins JM, Magee TR, Galland RB. Phlegmasia caerulea dolens and venous gangrene. Br J Surg 1996;83:1923.

2) Chinsakchai K, Ten Duis K, Moll FL, de Borst GJ. Trends in management of phlegmasia cerulea dolens. Vasc Endovascular Surg 2011;45:5-14.

3) Lorimer JW, Semelhago LC, Barber GG. Venous gangrene of the extremities. Can J Surg 1994;37:379-384.

4) Mousa AY, AbuRahma AF. May-Thurner syndrome: update and review. Ann Vasc Surg 2013;27:984-995.

5) O'Sullivan GJ, Semba CP, Bittner CA, Kee ST, Razavi MK, Sze DY, et al. Endovascular management of iliac vein compression (May-Thurner) syndrome. J Vasc Interv Radiol 2000;11:823-836.

6) Kaltenmeier CT, Erben Y, Indes J, Lee A, Dardik A, Sarac T, et al. Systematic review of May-Thurner syndrome with emphasis on gender differences. J Vasc Surg Venous Lymphat Disord 2018;6:399-407.e4.

7) Meissner MH, Gloviczki P, Comerota AJ, Dalsing MC, Eklof BG, Gillespie DL, et al. Early thrombus removal strategies for acute deep venous thrombosis: clinical practice guidelines of the Society for Vascular Surgery and the American Venous Forum. J Vasc Surg 2012;55:1449-1462.

8) Enden T, Haig Y, Kløw NE, Slagsvold
CE, Sandvik L, Ghanima W, et al. Long-term outcome after additional catheter-directed thrombolysis versus standard treatment for acute iliofemoral deep vein thrombosis (the CaVenT study): a randomised controlled trial. Lancet 2012;379:31-38.

9) Mewissen MW, Seabrook GR, Meissner MH, Cynamon J, Labropoulos $\mathrm{N}$, Haughton SH. Catheter-directed thrombolysis for lower extremity deep venous thrombosis: report of a national multicenter registry. Radiology 1999;211:39-49.

10) Karthikesalingam A, Young EL, Hinchliffe RJ, Loftus IM, Thompson MM, Holt PJ. A systematic review of 
percutaneous mechanical thrombectomy in the treatment of deep venous thrombosis. Eur J Vasc Endovasc Surg 2011;41:554-565.

11) Mahnken $\mathrm{AH}$, Thomson $\mathrm{K}$, de Haan M, O'Sullivan GJ. CIRSE standards of practice guidelines on iliocaval stenting. Cardiovasc Intervent Radiol 2014;37:889-897.

12) Neglén P, Hollis KC, Olivier J, Raju S. Stenting of the venous outflow in chronic venous disease: long-term stent-related outcome, clinical, and hemodynamic result. J Vasc Surg 2007;46:979-990.

13) Berger A, Jaffe JW, York TN. Iliac compression syndrome treated with stent placement. J Vasc Surg 1995;21:510-514.

14) Funatsu A, Anzai $H$, Komiyama K, Oi $\mathrm{K}$, Araki H, Tanabe Y, et al. Stent im- plantation for May-Thurner syndrome with acute deep venous thrombosis: acute and long-term results from the ATOMIC (AcTive stenting for MayThurner lliac Compression syndrome) registry. Cardiovasc Interv Ther 2019;34:131-138.

15) Al-Hakim RA, Boscanin A, Prosser DD, Kaufman JA. Management of phlegmasia cerulea dolens with percutaneous mechanical thrombectomy. Cardiovasc Intervent Radiol 2020;43:1398-1401.

16) O'Connor CT, Murray PC, Ryan MF, Byrne D. A case of phlegmasia cerulea dolens as a result of May-Thurner syndrome. QJM 2020;113:419-420.

17) Galanakis N, Kontopodis N, Peteinarakis 1 , Kehagias E, loannou CV, Tsetis D. Direct stenting in patients with acute lower limb arterial occlusions: immediate and long-term results. Cardiovasc Intervent Radiol 2017;40:192201.

18) Massi l, Zamboni P. What are the ideal characteristics of a venous stent? Veins Lymphat 2021;10:9739.

19) de Wolf MA, de Graaf R, Kurstjens RL, Penninx S, Jalaie H, Wittens $\mathrm{CH}$. Short-term clinical experience with a dedicated venous nitinol stent: initial results with the sinus-venous stent. Eur J Vasc Endovasc Surg 2015;50:518-526.

20) Ye K, Lu X, Li W, Huang $Y$, Huang $X$, $\mathrm{Lu} \mathrm{M}$, et al. Long-term outcomes of stent placement for symptomatic nonthrombotic iliac vein compression lesions in chronic venous disease. J Vasc Interv Radiol 2012;23:497-502. 